
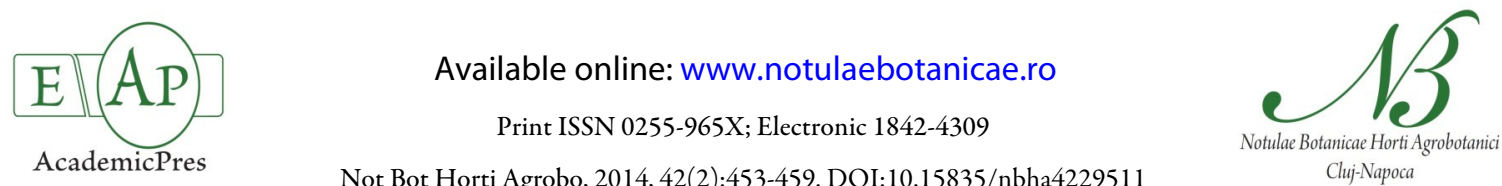

\title{
Genetic Diversity of High and Low Molecular Weight Glutenin Subunits in Algerian Aegilops geniculata
}

\author{
Asma MEDOURI, Inès BELLIL, Douadi KHELIFI* \\ Constantine 1 University, Faculty of Natural Sciences and Life, Laboratory of Genetic, Biochemistry and Plant Biotechnology, 25000, Constantine, Algeria; \\ asmabioch@yahoo.fr; bines07@yahoo.fr; dkhelifi@yahoo.fr(*correspondingauthor)
}

\begin{abstract}
Aegilops geniculata Roth is an annual grass relative to cultivated wheat and is widely distributed in North Algeria. Endosperm storage proteins of wheat and its relatives, namely glutenins and gliadins, play an important role in dough properties and bread making quality. In the present study, the different alleles encoded at the four glutenin loci (Glu-M1, GluU1, Glu-M3 and Glu-U3) were identified from thirty five accessions of the tetraploid wild wheat A. geniculata collected in Algeria using Sodium dodecyl Sulfate - Polyacrylamide Gel Electrophoresis (SDS-PAGE). At Glu-M1 and Glu-U1 loci, encoding high molecular weight glutenin subunits (HMW-GS) or A-subunits, 15 and 12 alleles were observed respectively, including one new subunit. B-Low molecular weight glutenin subunits zone (B-LMW-GS) displayed a far greater variation, as 28 and 25 alleles were identified at loci Glu-M3 and Glu-U3 respectively. Thirty two subunits patterns were revealed at the C subunits- zone and a total of thirty four patterns resulted from the genetic combination of the two zones (B-and C-zone). The wide range of glutenin subunits variation (high molecular weight glutenin subunits and low molecular weight glutenin subunits) in this species has the potential to enhance the genetic variability for improving the quality of wheat.
\end{abstract}

Keywords: alleles, electrophoresis, goatgrass, glutenins, polymorphism

\section{Introduction}

A. geniculata is an annual, autogamous, allotetraploid jointed goatgrass deriving from natural hybridization between Aegilops comosa $(2 n=2 x=14, \mathrm{MM})$ and Aegilops umbellulata $(2 n=2 x=14$, UU) (Kilian et al., 2011; Van Slageren, 1994). This tetraploid species is particularly interesting in wheat quality improvement especially as a source of resistance to various diseases, pests, drought and salinity (Zaharieva et al., $2001 \mathrm{a}, \mathrm{b}$ ).

The endosperm proteins of cultivated wheat, namely glutenins are important components of gluten which provides the unique viscoelastic properties indispensible for bread-making (Payne, 1987). Glutenins are aggregates of high molecular weight glutenin subunits (HMW-GS) or Asubunits and low molecular weight glutenin subunits (LMW-GS) or B-, C- and D-subunits, linked together by inter-molecular disulphide bonds. On SDS-PAGE, and according to known amino acid sequences, the group of Asubunits shows molecular mass ranging from 65.000 to 90.000 (Lagrain et al., 2013). The group of $\mathrm{B}$ subunits is slower moving with apparent molecular weight of about 40.000 to 50.000 comparing to $\mathrm{C}$-subunits which ranges from 30.000 to 40.000 . Thus the A-, B- and C-designations are based on their relative mobilities on SDS-PAGE (slower to faster, respectively) (Gianibelli $e t$ al., 2001; Masci et al.,
1993). The D-subunits group, migrating slightly slower than B-group was presumably called D because it was discovered after the assignment of $\mathrm{A}, \mathrm{B}$ and $\mathrm{C}$ designations (Payne and Corfield, 1979). The HMW-GS are encoded by the complex Glu-1 loci present on the long arm of group 1 homoeologous chromosomes and are further subdivided into high molecular $\mathrm{x}$-type and low molecular $\mathrm{y}$-type subunits tightly linked pairs of genes encoding one $\mathrm{x}$-type and one y-type subunits. The LMW-GS account for about one-third of the seed protein and roughly $60 \%$ of glutenin proteins, are mostly encoded by genes located on the short arms of the group 1 chromosomes, which form a multigene family (Glu-3 loci), closely linked to the Gli-1 loci containing genes encoding omega- and gamma-gliadins. This fraction of seed proteins play an important role in determining dough properties and the quality of wheat food products (Branlard et al., 2001; Gupta et al., 1991, 1994; Howitt et al., 2006). The copy number of LMW-GS genes in wheat was estimated to range from 10-20 to 30-40 (D'Ovidio and Masci, 2004; Liu et al., 2010; Zhang et al., 2013). Many studies were carried out to analyse the genetic diversity of Aegilops geniculata which is considered as source of new genes (Bandou et al., 2009; Monte et al., 1999; Zhang et al., 1996). However, no research has been conducted on the allelic composition of HMW and 
LMW-GS in this species. The objective of this study was to determine the allelic composition at four glutenins loci $(G l u$ $M 1, G l u-U 1, G l u-M 3$ and $G l u-U 3$ ) encoding for HMW (Asubunits) and LMW-GS (B- and C-subunits) within $A$. geniculata accessions.

\section{Materials and methods}

\section{Plant material}

Thirty five accessions of Aegilops geniculata originating from Algeria were analyzed for high and low molecular weight glutenin subunits. This collection was sampled throughout North of Algeria after seed maturation. Diploid progenitors; Aegilops comosa and Aegilops umbellulata kindly provided by the International Center for Agricultural Research in the Dry Areas (ICARDA) were used to assign components of the tetraploid species to $\mathrm{M}$ or $\mathrm{U}$ genomes.

\section{Protein analysis}

Glutenins were extracted from the brush of kernels following the sequential procedure described by Singh et al. (1991) and were separated using SDS-PAGE according to the method of Singh et al. (1991).

\section{Nomenclature}

To allocate components of the HMW-GS and B- LMWGS patterns of the tetraploid species $A$. geniculata to $\mathrm{U}$ or $\mathrm{M}$ genomes, comparison of glutenins electrophoretic patterns between $A$. geniculata accessions and diploid progenitors' accessions ( $A$. comosa and $A$. umbellulata) was made.

The nomenclature of subunits and alleles of HMW-GS (Glu-1 locus) corresponds to the terminology of RodriguezQuijano et al. (2001), prefixed with the letter $M$ for bands from A. comosa and the letter $\mathrm{U}$ for bands from $A$. umbellulata. For LMW-GS (B-and C-subunits), the different bands were numbered according to their mobility order in SDS-PAGE and a nomenclature was proposed for B-subunits.

\section{Results}

During the initial analysis of HMW-GS and LMW-GS patterns from different accessions of the collection, it was realized that almost all of them were unique in their overallbanding patterns; and only one accession was found to consist of two biotypes of HMW-GS with respect to LMW-GS. All the genes for B-glutenin subunits are located on the short arms of group 1 chromosomes of wheat and relatives (Gupta, 1991). However, Gupta et al. (1995) found a few C type LMW-GS that are controlled by genes on other chromosome arms. Furthermore, another study has confirmed that two Csubunits are encoded by genes located at the short arm of the chromosome 6 (Felix et al., 1996). For these reasons, the allelic variation of LMW-GS patterns of $A$. geniculata accessions at the $G l u-3$ loci has been done considering only the B-subunits. For C-subunits, a typology has been carried out. Thus, to simplify their analysis, LMW-GS patterns were studied separately for B-and C-subunits.
Characterization of $A$-, B- and $C$ - glutenin subunits patterns

Important variability was observed for A-glutenin subunits. A total of twenty nine patterns were revealed from the analysis of different $A$. geniculata accessions. Twenty three patterns were specific to one accession each. All of the accessions analyzed possessed four subunits (Fig. 1) in the A-zone except two accessions (G4 and G32 corresponding to pattern 4 and pattern 27 respectively) which showed three subunits each (Fig. 2). Likewise, extensive variation in the number and relative mobility of B-subunits was observed in the material under analysis. Considering the two loci together (Glu-M3 and Glu-U3), up to thirty three different patterns were identified and are shown in Figure 3.A. The number of glutenin subunits present in the B-zone varied from two to seven (patterns 9 and 18: Fig. 3.A), and some B-subunits appear to be common in several patterns. A clear resolution was obtained and it was possible to make a diagram of all the proteins present in the C-zone. Thirty two different patterns in C-subunits were observed, representing a slightly lower variation than that observed in $\mathrm{B}$-subunits. The number of $\mathrm{C}$-subunits varied between one (pattern 6 Fig. 3.B) and six subunits, and only one accession (G12) presented six C-subunits (pattern 32: Fig. 3.B), while $82.86 \%$ of the collection had three to five $\mathrm{C}$-subunits. Accessions with the same C-subunits and different Bsubunits were observed (G11and G34). Considering the two zones together (B- and C-subunits), each accession exhibited from four to eleven LMW-GS, which result in thirty four different patterns.

\section{Allelic variation at Glu-1 loci}

Twenty seven alleles were detected at the two loci together. Fifteen alleles were found at the Glu-MI locus (Tab. 1). Alleles Glu-MIl and Glu-MIi were the most frequent with $20.00 \%$ and $14.29 \%$ respectively, followed by allele Glu-M1h at a frequency of $11.46 \%$. Alleles Glu-M1e and Glu-MIn have been detected each in $8.57 \%$ of the collection. The allele Glu-MIm coding for subunit 7 only and alleles Glu-MIc and Glu-MId were found in two accessions each. The seven remaining alleles (Glu-Mla,

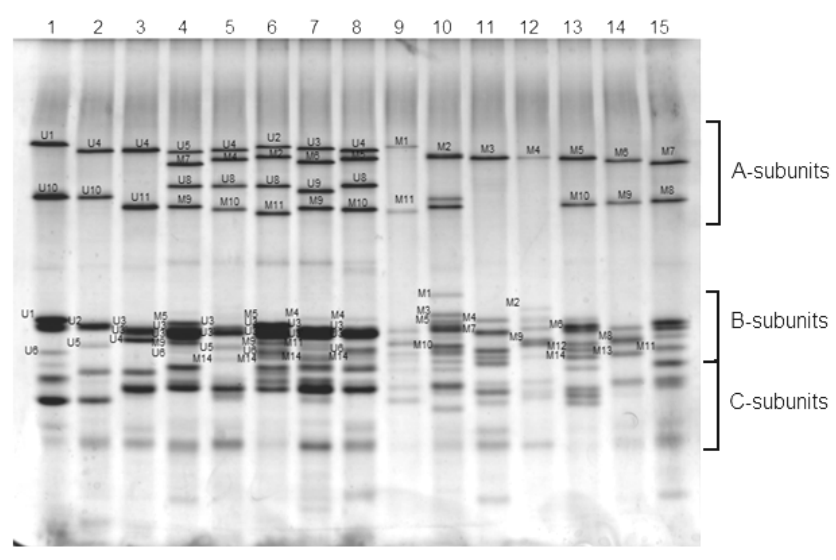

Fig. 1. SDS-PAGE patterns of HMW and LMW in some accessions of Aegilops geniculata collected in Algeria: 4: G1; 5: G2; 6: G3; 7: G15 and 8: G23; 1, 2 and 3: A. umbellulata; 9, $10,11,12,13,14$ and $15:$ A. comosa 
455

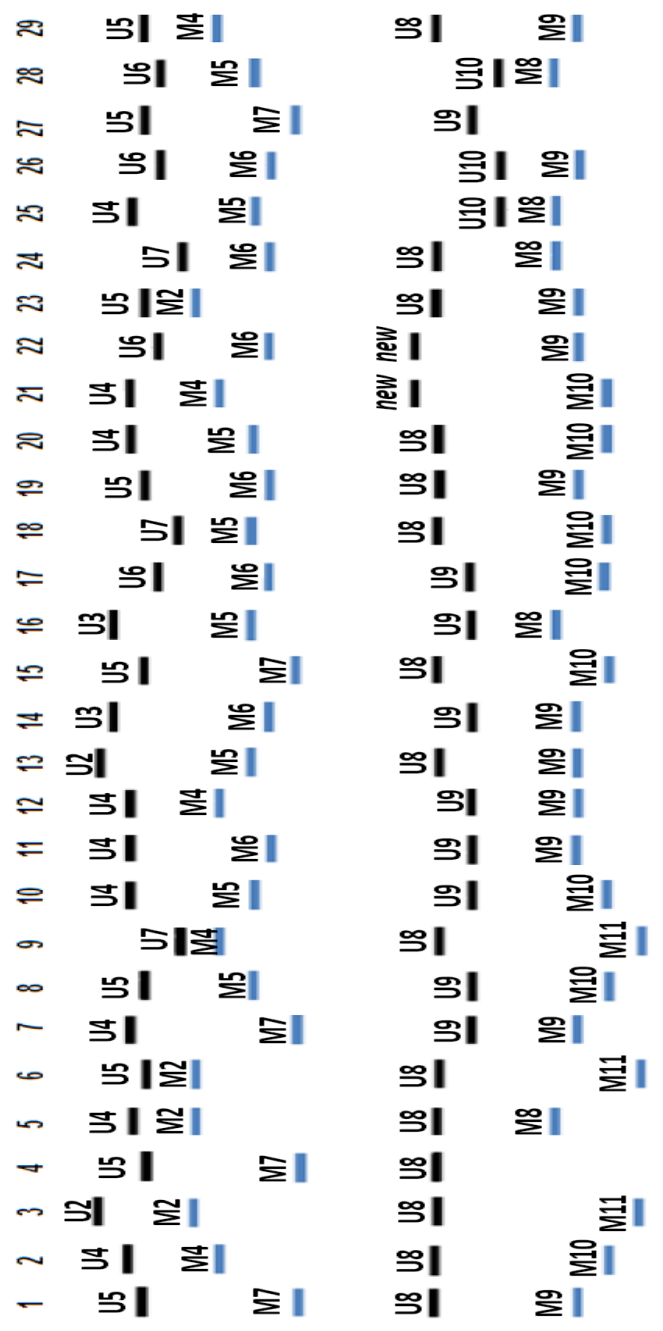

Fig. 2. Diagrammatic representation of HMW-GS patterns found in Aegilops geniculata from Algeria
Glu-M1b, Glu-M1f, Glu-M1g, Glu-M1 j, Glu-M1k and Glu-M1o) were rare in the investigated material with $2.86 \%$. At the $G l u-U 1$ locus, up to twelve alleles were detected including a subunit which has not been previously described, characterized by a slightly slower mobility comparing to subunit 8 (Fig. 4.A), this novel subunit identified at the Glu-U1 locus (designated new) was detected in two accessions (G26 and G27). The Glu-U1f allele (encoding the subunit pair 5+8) and Glu-U1d (subunits 4+9) were predominant with frequencies of $25.71 \%$ and $20.00 \%$, respectively. Alleles Glu-UIa, Glu-U1c and $G l u-U 1 j$ were found in three accessions each. Alleles $G l u-U 1 b, G l u-U 1 g$ and Glu-U1i appeared in $5.71 \%$ of the collection each, whereas alleles Glu-Ule, Glu-U1b besides to alleles Glu-U1k and Glu-U1l coding for (4+ new) and (6+ new) respectively; were considered to be rare and have been detected in only one accession each (Tab. 2).

\section{Allelic variation at Glu-3 loci in zone B}

This set of $A$. geniculata germplasm displayed abundant allelic variation for B-LMW-GS. A total of 53 alleles were identified at the B-zone (Tab. 1), 28 at the Glu-M3 locus (Fig. 4 B) and 25 at Glu-U3 locus (Fig. 4 C). At the Glu-M3 locus, approximately all the alleles were detected with the same frequency $(2.86 \%)$ except alleles Glu-M3a, Glu-M3k, Glu-M3m, Glu-M3q and Glu-M3y coding respectively for (null form, 5+9, 7+13, 4+7+13 and 7+9+12+13) which were present with $5.71 \%$ each. Only Glu-M3d (encoding subunit 9) had a frequency of $8.57 \%$. At the Glu-U3 locus, eighteen alleles were detected in one accession each. Five alleles (Glu-U3b, Glu-U3d Glu-U3g, Glu-U3i and Glu$U 3 m$ ) were detected in two accession each (5.71\%). The most frequent allele ( Glu- $U 3 u$ coding for subunits $3+3$ ' +6$)$ was detected in four accessions (11.43\% of the collection), Glu-M3t (encoding subunit 3' alone) was less frequent with $8.57 \%$.

$\mathbf{A}$

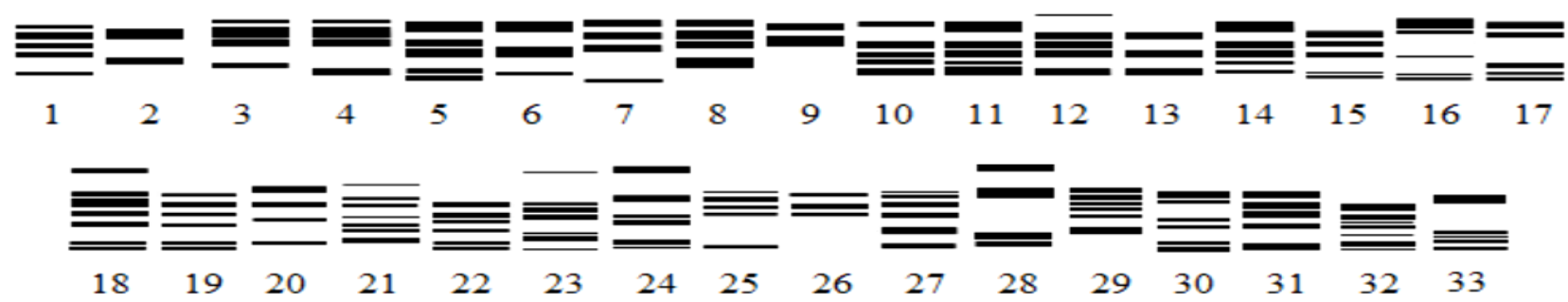

B

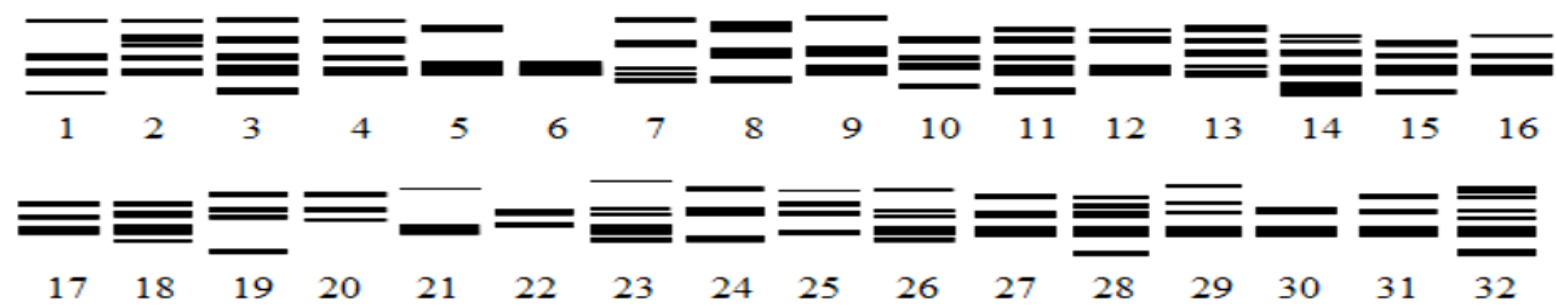

Fig. 3. Diagrammatic representation of (A): B-glutenin subunits patterns and (B): C-glutenin subunits patterns present in Aegilops geniculata accessions 
Tab. 1. Allelic composition of HMW and B-LMW glutenin subunits of Aegilops geniculata accessions

\begin{tabular}{|c|c|c|c|c|c|c|c|c|c|}
\hline Accessions & Province & Glu-MI & Allele & Glu-UI & Allele & Glu-M3 & Allele & Glu-U3 & Allele \\
\hline G1 & Annaba & $7+9$ & $n$ & $5+8$ & $f$ & $5+9$ & $k$ & $3+3^{\prime}+6$ & $u$ \\
\hline G2 & Guelma & $4+10$ & $d$ & $4+8$ & $a$ & $\mathrm{Nul}$ & $a$ & $3+5$ & $m$ \\
\hline G3 & Oum Bouaghi & $2+11$ & $c$ & $2+8$ & $b$ & $5+9$ & $k$ & $3+3^{\prime}+6$ & $u$ \\
\hline G4 & Batna 1 & 7 & $m$ & $5+8$ & $f$ & $7+13$ & $m$ & $2+4+6$ & $t$ \\
\hline G5 & Batna 2 & $2+8$ & $a$ & $4+8$ & $a$ & Nul & $a$ & $2+4+6$ & $t$ \\
\hline G6 & Batna 3 & $2+11$ & $c$ & $5+8$ & $f$ & $6+8+13$ & $r$ & 1 & $b$ \\
\hline G7 & Constantine 1 & $7+9$ & $n$ & $4+9$ & $d$ & $2+12$ & $f$ & $2+6$ & $k$ \\
\hline G8 & Constantine 2 & $5+10$ & $i$ & $5+9$ & $g$ & $4+6+7+11$ & u & $1+3$ & $g$ \\
\hline G9 & Constantine 3 & $4+11$ & $f$ & $7+8$ & $j$ & $8+9+12+13$ & $z$ & $1+2$ & $f$ \\
\hline G10 & Constantine 4 & $5+10$ & $i$ & $4+9$ & $d$ & $6+7+9+13$ & $w$ & 1 & $b$ \\
\hline G11 & Constantine 5 & $6+9$ & $l$ & $4+9$ & $d$ & $7+13$ & $m$ & $3+3^{\prime}+4+5+6$ & $y$ \\
\hline G12 & Constantine 6 & $5+10$ & $i$ & $4+9$ & $d$ & $10+12+13$ & $s$ & $2+5$ & $j$ \\
\hline G13 & Constantine 7 & $4+9$ & $e$ & $4+9$ & $d$ & $2+7+13$ & $o$ & $2+3^{\prime}+6$ & $s$ \\
\hline G14 & Constantine 8 & $5+9$ & $k$ & $2+8$ & $b$ & $4+13$ & $i$ & $1+3+3^{\prime}+5$ & $w$ \\
\hline G15 & Skikda & $6+9$ & $l$ & $3+9$ & $c$ & $4+11$ & $b$ & $3+3$ & $l$ \\
\hline G16 & Jijel & $7+10$ & $o$ & $5+8$ & $f$ & $2+6+7+11+13$ & $a a$ & $3+5$ & $m$ \\
\hline G17 & Mila 1 & $5+8$ & $b$ & $3+9$ & $c$ & $4+7+13$ & $q$ & $2+3$ & $i$ \\
\hline G18 & Mila 2 & $5+8$ & $b$ & $3+9$ & $c$ & $4+7+13$ & $q$ & $2+3$ & $i$ \\
\hline G19 & Mila 3 & $6+10$ & $g$ & $6+9$ & $b$ & 7 & $c$ & $1+3$ & $g$ \\
\hline G20 & Béjaia 1 & $5+10$ & $i$ & $7+8$ & $j$ & 11 & $e$ & $1+3+3$ & $p$ \\
\hline G21 & Béjaia 2 & $6+9$ & $l$ & $5+8$ & $f$ & $5+7$ & $j$ & Null & $a$ \\
\hline G22 & Bordj Bouariridj & $6+9$ & $l$ & $5+8$ & $f$ & 9 & $d$ & $1+3+5+6$ & $v$ \\
\hline G23 & Bouira & $5+10$ & $i$ & $4+8$ & $a$ & 4 & $b$ & $3+3^{\prime}+6$ & $u$ \\
\hline G24 & TiziOuzou & $7+9$ & $n$ & $5+8$ & $f$ & 9 & $d$ & $2+3^{\prime}+5+6$ & $x$ \\
\hline G25 & Blida & $6+9$ & $l$ & $4+9$ & $d$ & $3+9$ & $g$ & $3+3^{\prime}+6$ & u \\
\hline G26 & Médéa & $4+10$ & $d$ & $4+$ new & $k$ & 9 & $d$ & $3^{\prime}+6$ & $o$ \\
\hline G27 & Tissemsilt & $6+9$ & $l$ & $6+$ new & $l$ & $9+12$ & $n$ & $2+3^{\prime}+5$ & $r$ \\
\hline G28 & Ain Defla & $2+9$ & $b$ & $5+8$ & $f$ & $7+9+12+13$ & $y$ & $3^{\prime}$ & $d$ \\
\hline G29 & Chlef & $6+8$ & $j$ & $7+8$ & $j$ & $5+13$ & $l$ & $2+4+6$ & $t$ \\
\hline G30 & Relizane & $5+8$ & $b$ & $4+10$ & $e$ & $5+11+12+13$ & $v$ & 3 & $c$ \\
\hline G31 & Mascara & $6+9$ & $l$ & $6+10$ & $i$ & $7+9+12+13$ & $y$ & $1+3$ & $b$ \\
\hline G32 & Mostaganem & 7 & $m$ & $5+9$ & $g$ & $2+7+9+13$ & $t$ & $1+3+6$ & $q$ \\
\hline G33 & Sidi Belabbes & $5+8$ & $b$ & $6+10$ & $i$ & $4+6+9$ & $p$ & 6 & $e$ \\
\hline G34 & Saida & $4+9$ & $e$ & $4+9$ & $d$ & $3+5+6+9+10+12$ & $a b$ & $3^{\prime}$ & $d$ \\
\hline G35 & Tiaret & $4+9$ & $e$ & $5+8$ & $f$ & $6+7+10+13$ & $x$ & $3+6$ & $n$ \\
\hline
\end{tabular}

A

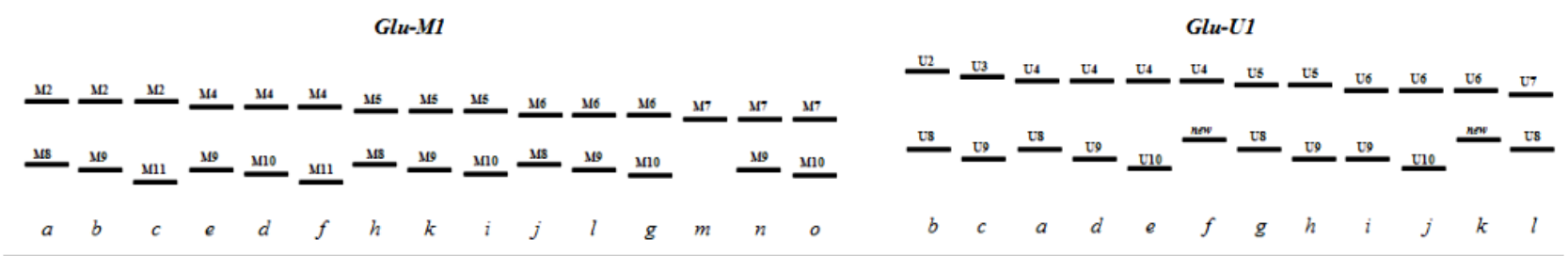

B

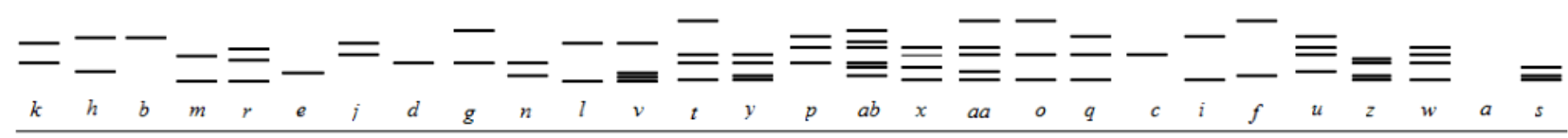

C

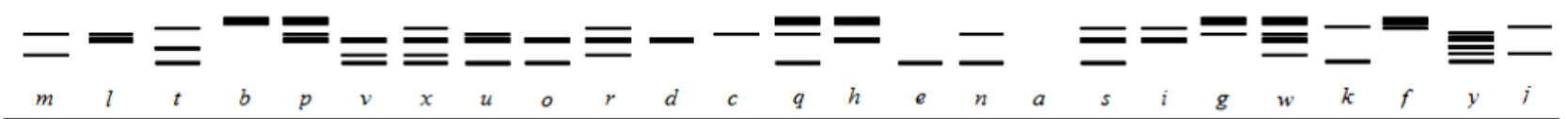

Fig. 4. Schematic representation of the mobility on SDS-PAGE of the different alleles encoded at (A): Glu-MI and Glu-U1 loci, (B): Glu-M3 and (C): Glu-U3 loci found in Aegilops geniculata accessions studied 
457

Tab. 2. Allele frequencies at HMW glutenin subunits and genetic index diversity at the Glu-M1 and Glu-U1 loci in Aegilops geniculata accessions

\begin{tabular}{|c|c|c|c|c|}
\hline Locus & Allele & Subunits & Frequency & $(\%)$ \\
\hline \multirow{16}{*}{$\begin{array}{c}\text { Glu- } \\
M I\end{array}$} & $a$ & $2+8$ & 1 & 2.86 \\
\hline & $b$ & $2+9$ & 1 & 2.86 \\
\hline & $c$ & $2+11$ & 2 & 5.71 \\
\hline & $e$ & $4+9$ & 3 & 8.57 \\
\hline & $d$ & $4+10$ & 2 & 5.71 \\
\hline & $f$ & $4+11$ & 1 & 2.86 \\
\hline & $b$ & $5+8$ & 4 & 11.42 \\
\hline & $k$ & $5+9$ & 1 & 2.86 \\
\hline & $i$ & $5+10$ & 5 & 14.29 \\
\hline & $j$ & $6+8$ & 1 & 2.86 \\
\hline & $l$ & $6+9$ & 7 & 20.00 \\
\hline & $g$ & $6+10$ & 1 & 2.86 \\
\hline & $m$ & 7 & 2 & 5.71 \\
\hline & $n$ & $7+9$ & 3 & 8.57 \\
\hline & $o$ & $7+10$ & 1 & 2.86 \\
\hline & H index & & & 0.90 \\
\hline \multirow{13}{*}{$\begin{array}{c}\text { Glu- } \\
\text { UI }\end{array}$} & $b$ & $2+8$ & 2 & 5.71 \\
\hline & $c$ & $3+9$ & 3 & 8.57 \\
\hline & $a$ & $4+8$ & 3 & 8.57 \\
\hline & $d$ & $4+9$ & 7 & 20.00 \\
\hline & $e$ & $4+10$ & 1 & 2.86 \\
\hline & $f$ & $5+8$ & 9 & 25.71 \\
\hline & $g$ & $5+9$ & 2 & 5.71 \\
\hline & $b$ & $6+9$ & 1 & 2.86 \\
\hline & $i$ & $6+10$ & 2 & 5.71 \\
\hline & $j$ & $7+8$ & 3 & 8.57 \\
\hline & $k$ & $4+$ new & 1 & 2.86 \\
\hline & $l$ & $6+n e w$ & 1 & 2.86 \\
\hline & $\mathrm{H}$ index & & & 0.86 \\
\hline
\end{tabular}

\section{Discussion}

In this study, we analyzed the variation of HMW and LMW-GS in a set of thirty five $A$. geniculata accessions. Concerning the Glu-1 loci, twenty seven alleles were detected; representing fifteen alleles at the Glu-MI locus and twelve alleles at the Glu-UI locus. This diversity is higher than that revealed in its diploid parents; $A$. comosa and $A$. umbellulata, where eleven and eight alleles were found at the Glu-M1 and Glu-U1 loci respectively (Rodriguez-Quijano et al., 2001). A total of nineteen HMW subunits were revealed, resulting in twenty nine subunits patterns, higher than that found by Bandou et al. (2009) who analyzed the variability within fourteen $A$. geniculata populations collected in North Algeria and detected sixteen HMW-GS bands which in combination generated twenty eight phenotypes. In our study, the Glu$M 1$ locus displayed the highest level of variation (45.71\%) comparing to the Glu-U1 locus (34.29\%), this finding was also observed by Rodriguez-Quijano et al. (2001), who revealed $33.3 \%$ and $27.6 \%$ for the Glu-MI and Glu-U1 loci respectively. Kozub et al. (2011) when analyzing a collection of $A$. biuncialis (UU MbMb) from Ukraine have noticed that the $G l u-M 1$ was more polymorphic (10 alleles at the $G l u-M 1$ locus and 8 alleles at the Glu-U1 locus). Moreover the genetic diversity index at the Glu-MI locus $(\mathrm{H}=0.90)$ was slightly higher than the Glu-U1 locus $(\mathrm{H}=0.86)$. HMW-GS alleles controlling the x-type subunit only were detected in two accessions (G04 and G32) at Glu-M1 locus (allele Glu-MIm). Alleles coding for the x-type subunit only were encountered in diploid and tetraploid Aegilops species (Kozub et al., 2011; Rodriguez-Quijano et al., 2001), common at the Glu-A1 locus and rare at the Glu-D1 in cultivated wheat (Fernández-Calvín and Orellana, 1990; Payne and Lawrence, 1983; Saponaro et al., 1995).

Extensive variation in the number and relative mobilities of the B-glutenin subunits was observed in $A$. geniculata; fifty three alleles were counted at the two loci for B-LMWGS which in combination result in thirty three subunits patterns. This variability was slightly higher than that observed in Aegilops tauschii for the same zone by Gianibelli et al. (2002), who found thirty patterns, however it was lower than that revealed by Caballero et al. (2004) who detected 46 patterns in spelt wheat. Moreover, variation found in our study was higher than that revealed in Algerian durum and bread wheat (Bellil et al., 2012, 2014 a, b; Cherdouh et al., 2005), and much higher than that reported in other collections of durum wheat (Nieto-Taladriz et al., 1997; Ruiz et al., 1998) and in some wheat cultivars (Branlard et al., 2003; Gupta and Shepherd, 1990). This indicates the importance of the collection under analysis as a source of new genes for LMW-GS. In some cases, difference in B-LMW patterns was limited in only one subunit (band); for example, accessions G1 and G25 (pattern 1 and 12: Fig. 3.A), this could be explained by the silencing of the corresponding gene. Quantitative variations were frequently detected, thus strongly stained bands could indicate the presence of this gene in a high copy number or the presence of one subunit or more with the same apparent molecular weight. In some studies, the high gene copy number was attributed to be the cause of the high level of HMW-GS 7 coded by Glu-B1 locus in a landrace and a cultivar of hexaploid wheat (D'Ovidio et al., 1997; Lukow et al., 1992).

For the $\mathrm{C}$ zone, thirty two patterns were found, much higher than that detected by Caballero et al. (2004) in spelt wheat; however Gianibelli et al. (2002) revealed forty three patterns in a collection of $A$. tauschii, much higher than that we found. In contrast to our study, Gianibelli et al. (2002) observed more polymorphism for the C-LMW-GS zone than the B-LMW-GS zone. Like in B-subunits zone, differences between some patterns in the $\mathrm{C}$ zone lay only in one subunit as revealed in pattern 3 and pattern 11 (Fig. 3.B). Differently to B-subunits patterns, some patterns could be distinguished only by the presence or the absence of only one specific subunit, for example patterns 30 and 31 differed by the presence of an extra subunit in the latter pattern (Fig. 3.B). These results provide more evidence that $B$ and $C$ subunits could be encoded by two separate gene subfamilies although they are closely linked (Gupta and Shepherd, 1990). The presence of accessions with identical $\mathrm{B}$-subunits patterns and different $\mathrm{C}$-subunits patterns and vice-versa reinforce earlier studies suggesting that some Csubunits are encoded by genes at different loci (Felix et al., 1996; Gupta and Shepherd, 1993). The important number of band patterns found in this study (thirty four, thirty two and thirty four patterns for; B-subunits, C-subunits and Band C-subunits together respectively) is higher than that reported in durum and bread wheat studies giving 
possibilities to enhance dough properties by introducing new genes of quality.

In conclusion, this work shows that Algerian $A$. geniculata exhibited a wide range of variation in HMW-GS and LMW-GS, including new subunits and alleles, this indicates that this species is an important source for increasing genetic variability in cultivated wheat and those alleles are likely to be useful via wheat breeding programs to provide improvement to flour properties.

\section{References}

Bandou H, Rodriguez-Quijano M, Carrillo JM, Branlard G, Zaharieva M, Monneveux P (2009). Morphological and genetic variation in Aegilops geniculata from Algeria. Plant Syst Evol 277:85-97.

Bellil I, Chekara Bouziani M, Khelifi D (2012). Genetic diversity of high and low molecular weight glutenin subunits in Saharan bread and durum wheats from Algerian oases. Czech J Genet Plant 48:23-32.

Bellil I, Hamdi O, Khelifi D (2014a). Diversity of five glutenin loci within durum wheat (Triticum turgidum L. ssp. durum (Desf.) Husn.) germplasm grown in Algeria. Plant Breeding 133:179-183.

Bellil I, Hamdi O, Khelifi D (2014b). Allelic variation in Glu-1 and Glu-3 loci of bread wheat (Triticum aestivum ssp. aestivum L. em. Thell.) germplasm cultivated in Algeria. Cereal Res Commun doi:10.1556/CRC.2014.0004.

Branlard G, Dardevet M, Amiour N, Igrejas G (2003). Allelic diversity of HMW and LMW glutenin subunits and omega gliadins in French bread wheat (Triticum aestivum L.). Genet Resour Crop Ev 50:669-679.

Branlard G, Dardevet M, Saccomano R, Lagoutte F, Gourdon J (2001). Genetic diversity of wheat storage proteins and bread wheat quality. Euphytica 119: 59-67.

Caballero L, Martín LM, Alvarez JB (2004). Genetic variability of the low-molecular-weight glutenin subunits in spelt wheat (Triticum aestivum ssp. spelta L. em Thell.). Theor Appl Genet 108:914-919.

Cherdouh A, Khelifi D, Carrillo JM, Nieto-Taladriz MT (2005). The high and low molecular weight glutenin polymorphism of Algerian durum wheat landraces and old cultivars. Plant Breeding 124:338-342.

D'Ovidio R, Masci S (2004). The low-molecular-weight glutenin subunits of wheat gluten. J Cereal Sci 39:321-339.

D’Ovidio R, Masci S, Porceddu E, Kasarda DD (1997) Duplication of the $B x 7$ high-molecular-weight glutenin and subunit gene in bread wheat (Triticum aestivum L.) cultivar Red River 68. Plant Breeding 116:525-531.

Felix I, Martinant JP, Bernard M, Bernard S, Branlard G (1996). Genetic characterization of storage proteins in a set of Flderived haploid lines in bread wheat. Theor Appl Genet 92:340-346.
Fernández-Calvín B, Orellana J (1990). High molecular weight glutenin subunit variation in the Sitopsis section of Aegilops. Implications for the origin of the B genome of wheat. Heredity 65:455-463.

Gianibelli MC, Gupta RB, Lafiandra D, Margiotta B, MacRitchie F (2001). Polymorphism of High Mr Glutenin Subunits in Triticum tauschii: Characterisation by Chromatography and Electrophoretic Methods. J Cereal Sci 33:39-52.

Gianibelli MC, Wrigley CW, MacRitchie F (2002). Polymorphism of Low Mr Glutenin Subunits in Triticum tauschii. J Cereal Sci 35:277-286.

Gupta PK (1991). Chromosome engineering in plants: genetics, breeding, evolution, p. 243-262. In: Gupta PK, Tsuchyia T (Eds.). Cytogenetics of Wheat and its Close Wild RelativesTriticum and Aegilops. Elsevier, New York.

Gupta RB, Bekes F, Wrigley CW (1991). Prediction of physical dough properties from glutenin subunit composition in bread wheats - correlation studies. Cereal Chem 68:328-333.

Gupta RB, Paul JG, Cornish GB, Palmer GA, Bekes F, Rathjen AJ (1994). Allelic variation at glutenin subunit and gliadin loci, Glu-1, Glu-3 and Gli-1, of common wheat. I. Its additive and interaction effects on dough properties. J Cereal Sci 19:9-17.

Gupta RB, Popineaut P, Lefebvre J, Cornect M, Lawrence GJ, MacRitchie F (1995). Biochemical Basis of Flour Properties in Bread Wheats. II. Changes in Polymeric Protein Formation and Dough/Gluten Properties Associated with the Loss of Low Mr or High Mr Glutenin Subunits. J Cereal Sci 21:103116.

Gupta RB, Shepherd KW (1990). Two-step one-dimensional SDS-PAGE analysis of LMW subunits of glutelin. 2. Genetic control of the subunits in species related to wheat. Theor Appl Genet 80:183-187.

Gupta RB, Shepherd KW (1993). Production of multiple wheatrye 1 RS translocation stocks and genetic analysis of LMW subunits of glutenin and gliadins in wheat using these stocks. Theor Appl Genet 85:719-728.

Howitt CA, Gale KR, Juhász A (2006). Diagnostic markers for quality, p. 333-361. In: Wrigley CW, Békés F, Bushuk W (Eds.). Gliadin and glutenin. The unique balance of wheat quality. MN: AACCI Press, St Paul.

Kilian B, Mammen K., Millet E, Sharma R, Graner A, Salamini F, Hammer K, Özkan H (2011). Aegilops, p. 1-76. In: Chittaranjan Kole (Ed.). Wild Crop Relatives: Genomic and Breeding Resources Cereals. Springer Heidelberg Dordrecht, New York.

Kozub NA, Sozinova IA, Xyniasc IN, Sozinov AA (2011). Allelic Variation at High Molecular Weight Glutenin Subunit Loci in Aegilops biuncialis Vis. Russ J Genet 47:1216-1222.

Lagrain B, Brunnbauer M, Rombouts I, Koehler P (2013). Identification of Intact High Molecular Weight Glutenin Subunits from the Wheat Proteome Using Combined Liquid Chromatography-Electrospray Ionization Mass Spectrometry. Plos One 8:1-10. 
459

Liu L, Ikeda TM, Branlard G, Peña RJ, Rogers WJ, E Lerner S, Kolman MA, Xia X, Wang L, Ma W, Appels R, Yoshida H, Wang A, Yan Y, He Z (2010). Comparison of low molecular weight glutenin subunits identified by SDS-PAGE, 2-DE, MALDI-TOF-MS and PCR in common wheat. BMC Plant Biol 10:1-24.

Lukow OM, Forsyth SA, Payne PI (1992). Over-production of HMW glutenin subunits coded on chromosome $1 \mathrm{~B}$ in common wheat, Triticum aestivum. Journal of Genetics and Breeding 46:187-192.

Masci S, Lafiandra D, Porcedu E, Lew EJL, Peggy Tao H, Kasarda DD (1993). D-glutenin subunits: N-Terminal sequences and evidence for the presence of cysteine. Cereal Chem 70(5):581585.

Monte JV, Casanova C, Soler C (1999). Genetic variation in Spanish populations of the genus Aegilops revealed by RAPDs. Agronomie 19:419-427.

Nieto-Taladriz MT, Ruiz M, Martínez MC, Vázquez JF, Carrillo JM (1997). Variation and classification of B low-molecularweight glutenin subunit alleles in durum wheat. Theor Appl Genet 95:1155-1160.

Payne PI (1987). Genetic of wheat storage proteins and the effect of allelic variation on bread-making quality. Ann Rev Plant Physio 38:141-153.

Payne PI, Corfield KG (1979). Subunit composition of wheat glutenin protein isolated by gel filtration in a dissociating medium. Planta 145:83-88.

Payne PI, Lawrence GJ (1983). Catalogue of alleles for the complex gene loci Glu-A1, Glu-B1and Glu-D1which code for the high-molecular-weight subunits of glutenin in hexaploid wheat. Cereal Res Commun 11:29-35.

Rodriguez-Quijano M, Nieto-Taladriz MT, Carrillo JM (2001). Polymorphism of high molecular weight glutenin subunits in three species of Aegilops. Genet Resour Crop Ev 48:599-607.
Ruiz M, Vázquez JF, Carrillo JM (1998). Study of glutenins and gliadins variability in Spanish landraces and primitive cultivars of durum wheat (in Spanish). Investigación Agraria Producción y Protectión Vegetales 13:291-305.

Saponaro C, Pogna NE, Castagna R, Pasquini M, Cacciatori P, Redaelli R (1995). Allelic variation at the Gli-A1 $1^{m}, G l i-A 2^{m}$ and $G l u-A I^{m}$ loci and bread making quality in diploid wheat Triticum monococcum. Genet Res Camb 66:127-137.

Singh NK, Shepherd KW, Cornich GB (1991). A simplified SDSPAGE procedure for separating LMW subunits of glutenin. J Cereal Sci 14:203-208.

Van Slageren MW (1994). Wild Wheats: a monograph of Aegilops L. and Amblyopyrum (Jaub. \& Spach) Eig (Poaceae). Wageningen Agricultural University Papers. Wageningen, The Netherlands, $513 \mathrm{p}$.

Zaharieva M, Gaulin E, Havaux M, Acevedo E, Monneveux P (2001a). Drought and heat responses in the wild wheat relative Aegilops geniculata Roth: potential interest for wheat improvement. Crop Sci 41:1321-1329.

Zaharieva M, Monneveux P, Henry M, Rivoal R, Valkoun J, Nachit MM (2001b). Evaluation of a collection of wild wheat relative Aegilops geniculata Roth and identification of potential sources for useful traits. Euphytica 119:33-38.

Zhang X, Liu D, Zhang J, Jiang W, Luo G, Yang W, Sun J, Tong Y, Cui D, Zhang A (2013). Novel insights into the composition, variation, organization, and expression of the low-molecular-weight glutenin subunit gene family in common wheat. J Exp Bot 64 (7):2027-40.

Zhang XY, Wang RC, Dong YS (1996). RAPD polymorphisms in Aegilops geniculata Roth (Ae. ovata auct. non L.). Genet Resour Crop Ev 43(5):429-433. 